\title{
Hegels Kritik der bürgerlichen Gesellschaft als Beitrag zur Anthropologie des Politischen
}

\author{
ANDRZEJ PRZYLEBSKI \\ Univ. Poznan
}

Georg Friedrich Wilhelm Hegel gehörte zweifelsohne zu den größten Figuren der abendländischen Philosophiegeschichte. Seine umfangreiche, allumfassende und zugleich logisch-begrifflich so anspruchsvolle Philosophie lädt förmlich zu immer neuen Versuchen ein, sie zu ergründen, zu verstehen und für andere zugänglicher zu machen. Hegel wurde für viele zu einer Forschungsdisziplin, in der sie sich ausweisen können, als akademische Lehrer und Forscher. Selten jedoch münden diese Untersuchungen in einem Versuch, die untilgbare Aktualität seines Denkens unterm Beweis zu stellen. Dies zu versuchen gilt heute als kaum vertretbar. Denn auch wenn wir bereit wären, Hegel in der Rolle des „Denkers der Moderne“ unter Umständen zu akzeptieren, so stünde der Aktualitätsthese bezüglich seiner Philosophie möglicherweise die einfache Feststellung, dass wir jetzt doch nicht in der Moderne, sondern in der Postmoderne leben. In einer Zeit also, für die Nietzsche maßgeblicher zu sein scheine als der „obsolet gewordene“ Hegel.

Im Unterschied zur solchen rein philosophiegeschichtlichen Lektüre Hegels will der vorliegende Text für die These über die wirkliche Aktualität eines Teils der Philosophie Hegels plädieren. Und es ist kein unwichtiger Teil, denn es geht um den Kern seiner Sozialphilosophie. Die Frage, die ich mir hier stelle, lautet: Ist Hegels Denken über eine gute Gesellschaft, ist sein Bild so einer, modernen (und nicht vor- oder postmodernen) Gesellschaft noch tragend, oder gehört es in die Archiven der Philosophiegeschichte. Die Antwort auf diese Frage hängt nicht nur vom Vergleich zweier Gesellschaftsbilder: des Hegelschen und des heutigen, ab. Sie hängt auch und vor allem von einem Bild der Gesellschaft ab, das wir als Ideal bzw. Optimal annehmen, einer Gesellschaft, derer Verwirklichung wir als erstrebenswert empfinden.

So ein Bild ist ohne weiteres mit der Anthropologie verbunden. Zwar nicht mit der, die wir beispielsweise in Hegels Enzyklopädie der philosophischen 
Wissenschaften finden können, wohl aber mit der Anthropologie des Politischen, die erst im 20. Jahrhundert entstanden ist und die wir solchen Denkern, wie Arnold Gehlen oder Hannah Arendt verdanken. In ihr geht es weniger um die begriffliche Fassung der subtilen Übergangs vom bloß Animalischen zum Menschlichen (wenn auch nur auf der niedrigsten Stufe der Menschenentwicklung). Es geht ihr vielmehr um Ausarbeitung und Hervorhebung der in der tierischen Welt abwesenden Züge des menschlichen Wesens, die den Menschen die Entstehung der Gesellschaft, die Bildung der Kultur, die Einbeziehung der Tradition und der Geschichte zu einer Not machten. In diesem Sinne versteht sich die vorliegende Untersuchung als Beitrag zur Anthropologie des Politischen, und zwar als ein solcher, der für die Aktualität der Hegelschen Gesellschaftsideal - in seinen Grundzügen, nicht in Details - plädiert.

\section{Hegels Auffassung DeR BÜRgerlichen GeSEllschaft}

Der Begriff der bürgerlichen Gesellschaft avancierte in der zweiten Hälfte des vorigen Jahrhunderts zum Hauptbegriff der politischen Theorien. G.F.W. Hegel gilt zu recht als einer der wichtigsten Theoretiker des Politischen, die zu Entwicklung und Statuierung dieses Begriffs maßgeblich beigetragen haben. Dabei wird oft übersehen, dass seine praktische Philosophie eine profunde Kritik der bürgerlichen Gesellschaft beinhaltet, und zwar die Kritik nicht nur im philosophischen, sondern auch im üblichen, alltäglichen Sinne des Wortes. Im meinem Beitrag möchte ich zeigen, wie Hegel bürgerliche Gesellschaft thematisiert, in welchem theoretischen Kontext er sie platziert und was daraus folgt, auch für das gegenwärtige Denken über die Rolle der bürgerlichen Gesellschaft in der Gesamtheit des sozialen und politischen Lebens, die geistige Gestaltung des Einzelmenschen einschließlich..

Hinter der berühmt gewordenen Feststellung Hegels, laut der ,das Vernünftige wirklich ist und das Wirkliche vernünftig“ steckt die Zurückweisung des ontologischen Abgrunds zwischen dem Reich des Seins und dem des Sollens. ${ }^{1}$ Indem er im Leben einer jeden Gesellschaft die Verwirklichung der Taten konstatiert, die im Sollen ihr Motiv haben, d.h. die aus dem Pflichtgefühl unternommen werden, bricht er mit den jenseitigen (noumenalen) Charakter einer ,wahren Moralität“ und verankert Moral in einem sowohl das individuelle wie das transzendentale Ich aufhebenden Standpunkt der Sittlichkeit. Für jede geistig entwickelte menschliche Gemeinschaft nimmt er die Existenz einer sie tragenden Substanz an, die ihre Kommunikation und Zusammenarbeit zugleich ermöglicht und bedingt. Diese geistige Substanz, die er mit dem Begriff „Sittlichkeit“ zum Ausdruck bringt, wirkt auf einem unbewussten Level, sie ist

1 Bekanntlich ist es der Ansatzpunkt der - nur partiell begründeten - Hegelchen Kritik an der Moralitätslehre von Kant. 
die geistige Grundlage der Kultur dieser Gemeinschaft. Diese Behauptung gilt insbesondere für die in Form einer Nation existierende Gesellschaft. ${ }^{2}$

Der oft als Vordenker des Liberalismus gedeutete Hegel war bekanntlich ein scharfer Gegner des Freiheitskonzeptes, das im sozialen Atomismus resultiert und zum abstrakten Individualismus führt. Unter anderem deswegen weist er die sozialen Vertragstheorien als Grundlage für die Erklärung der Entstehung des Staates zurück. So ein „Nullpunkt“, in dem die Individuen als Träger der abstrakten Rechte (z.B. der „ewigen Menschenrechte“) einen Vertag abschließen, sei eine abstrakte Konstruktion, die es in Wirklichkeit nie gegeben hat, denn jede Gesellschaft, jedes Volk, hat einen Entwicklungsweg hinter sich, die sein geistiges Leben mit Inhalten füllt. ${ }^{3}$ Diese Ansammlung der geschichtlichen Erfahrungen eines Volkes - Gadamer nannte sie in Wahrheit und Methode die $>$ Überlieferung < - zeichnet die Sittlichkeit bzw. die geistige Substanz dieses Volkes aus. ${ }^{4}$ Diese Substanz, in der eine Pflicht - zumindest als Handlungsmotiv - zugleich ein Sein hat, hat Wirklichkeit als Geist einer Nation, stellt Hegel in der Enzyklopädie fest. ${ }^{5}$ Die menschliche Person als eine denkende Intelligenz kennt diese Substanz als ihr eigenes Wesen. Eine Art Konkretisierung der so verstandenen ethischen Substanz ist das Entstehen und das Bestehen (SichManifestieren) solcher moralischen Werte (bzw. Einstellungen), wie Patriotismus, Gerechtigkeit, Vertrauen, Wohlwollen, Entgegenkommen, die für eine friedliche Koexistenz innerhalb eines Volkes unabdingbar sind

Die sittliche Substanz ist bei Hegel bekanntlich aus drei aufeinander unreduzierbaren und voneinander unableitbaren Elementen zusammengesetzt: der

2 Vgl. Hegel, Enzyklopädie der philosophischen Wissenschaften, in: Hegel, Werke in 20 Bänder, Suhrkamp, Frankfurt/M. 1986, B. 10, § 513: „Die Sittlichkeit is die Vollendung des objektiven Geistes, die Wahrheit des subjektiven und objektiven Geistes selbst“.

3 Die Interpretation des Sozialvertrags im Sinne eines Gedankenexperiments hätte das ablehnende Verdikt Hegels wahrscheinlich kaum gemildert, denn er sah die Nützlichkeit so eines künstlichen Konstruktes kaum ein.

4 Die Nähe der Hermeneutik Gadamers zur Gesellschafslehre Hegels ist an mehreren Stellen seines Hauptwerkes zu sehen. Vgl. z.B.: H.-G. Gadamer, Wahrheit und Methode, Ges. Werke B. 1, Mohr Siebeck, Tübingen 1986, S.360 ff. und 395 ff. oder „Über die Möglichkeiten einer philosophischen Ethik“, in: Gadamer, GW, B. 4, s. 175-188.

$5 \quad \mathrm{Vgl} .:$ Hegel, Enzyklopädie der philosophischen Wissenschaften, § 514: „Die frei sich wissende Substanz, in welcher das absolute Sollen ebenso sehr Sein ist, hat als Geist eines Volkes Wirklichkeit. Die abstrakte Diremtion dieses Geistes ist die Vereinzelung in Personen (...). Die Person aber weiß als denkende Intelligenz jene Substanz als ihr eigenes Wesen, hört in dieser Gesinnung auf, Akzidens derselben zu sein, schaut sie als ihren absoluten Endzweck in der Wirklichkeit sowohl als erreichtes Diesseits an, als sie denselben durch ihre Tätigkeit hervorbringt, aber als etwas, das vielmehr schlechthin ist; so vollbringt sie ohne die wählende Reflexion ihre Pflicht als das Ihrige und als Seiendes und hat in dieser Notwendigkeit sich selbst und ihre wirkliche Freiheit"“. 
Familie, der bürgerliche Gesellschaft und des Staates. ${ }^{6}$ In der modern gedachten, dennoch ausdrücklich monogam und heterosexuell gefassten Familie existiert der Geist unmittelbar, soz. in „,natürlicher Weise“. 7 Den Anfang der Familie bildet die in der Liebe und im Willen zur Fortpflanzung gründende Ehe, in der zwei Personen zur geistigen und rechtlichen Einheit gebracht werden. Die Verpflichtung der Ehe - sozusagen ihr gesellschaftlicher Auftrag - ist Kinderbesitz und Kindererziehung, damit sie neue Familien gründeten und so zur Entwicklung der bürgerlichen Gesellschaft bzw. der Nation beitragen könnten.

Anders als bei Kant ist die Ehe bei Hegel etwas mehr als nur ein zivilrechtlicher Vertrag, den man, wie jeden Vertrag, ohne ethische Skrupel annullieren darf. Ehe ist laut Hegel eine besondere, denn eine ethische Beziehung, in der wegen der Liebe als sie tragenden Verhältnis eine wahre Öffnung auf den anderen Mensch stattfindet. Die rechtlichen Verhältnisse sind hier vor allem stabilisierend, also: sekundär, äußerlich. Philosophische Relevanz erlangte diese Auffassung deshalb, weil hier gezeigt wird, dass die ursprüngliche, quasi-natürliche Moralität, die in der Familie entsteht, ihre Quelle in der Liebe und sie begleitenden Werten bzw. Einstellungen hat, wie Hilfsbereitschaft, Opferbereitschaft, gegenseitiges Vertrauen, Fürsorge, Verständigungswille. Sie lässt sich durch die Tätigkeiten seitens des Staates oder der bürgerlichen Gesellschaft nicht ersetzen.

All das wird evident, wenn Hegel feststellt, dass die Gestalt der ethischen Substanz, die von der bürgerlichen Gesellschaft (als Gemeinschaft vieler Familien) verwirklicht wird, ihre anfängliche sittliche Bestimmung verliert, denn das Ziel der Personen als Mitglieder der bürgerlichen Gesellschaft nicht die absolute Einheit, sondern die Eigenart (ein „Für-sich-Sein“) ist. In diesem Kontext spricht Hegel über eine „System der Atomistik“, was als Konstatierung der gesellschaftlichen Atomisierung verstanden werden kann, die durch den Vorrang der individuellen Interessen und Egoismen zustande kommt. Das ist der Beginn seiner Analysen der bürgerlichen Gesellschaft, die zugleich eine Kritik dieser Gesellschaft sind. Diese Kritik wird besonders in dem Moment aktuell, in dem die bürgerliche Gesellschaft die zwei sonstigen Bestandteile

6 Vgl.: Hegel, Enzyklopädie der philosophischen Wissenschaften, § 517: „Die sittliche Substanz ist: (a) als unmittelbarer oder natürlicher Geist - die Familie; (b) die relative Totalität der relativen Beziehungen der Individuen als selbstständigen Personen aufeinander in einer formellen Allgemeinheit - die bürgerliche Gesellschaft; (c) die selbstbewusste Substanz als der zu einer organischen Wirklichkeit entwickelte Geist - die Staatsverfassung“.

7 Vgl. Ebd. § 519: „Der Unterschied der natürlichen Geschlechter erscheint ebenso zugleich als ein Unterschied der intellektuellen und sittlichen Bestimmung. (...). Die substantielle Innigkeit macht die Ehe zu einem ungeteilten Bande der Personen, - zu monogamischer Ehe; die körperliche Vereinigung ist Folge des sittlich geknüpften Bandes. Die fernere Folge ist die Gemeinsamkeit der persönlichen u nd partikulären Interessen" 
der sittlichen Einheit einer Nation: die Familie und den Staat, gefährdet, indem sie ihre Rollen zu übernehmen versucht.

Die „Besonderheit der Personen“ bedeutet für Hegel vor allem die Mannigfaltigkeit der menschlichen Bedürfnisse, deren Befriedigung nur in einem durch die bürgerliche Gesellschaft initiierten System möglich sein wird, einem System, der auf der Arbeitsteilung basiert. ${ }^{8}$ Diese Entwicklung führt einerseits zum Wachstum des Reichtums (und der äußeren Freiheit), anderseits aber zur Reduktion der menschlichen Arbeit auf eine Art spezialisierten Geschicklichkeit, die etwas Unmenschlich-Mechanisches in sich hat. ${ }^{9}$ Hegel war ein scharfsinniger Beobachter des Kapitalismus seiner Zeit und fleißiger Leser der klassischen Werke der Nationalökonomie (Ricardo, Smith); er spürte die Bedrohungen, die mit der wachsenden Produktivität an der einen und der Entfremdung von der Arbeit auf der anderen Seite einhergehen. Er sieht, dass die Warenmasse, die in der kapitalistischen Wirtschaft entsteht, die Konsummöglichkeiten der Gesellschaft überschreitet, was zur Bedrohung ihrer ökonomischen Stabilität, sogar der Existenz, führen muss. Die einzige Lösung scheint der Export zu sein, notfalls zu den Kolonien, was in der wachsenden Kolonialisierung der Welt durch das kapitalistische Wirtschaftssystem resultieren muss. ${ }^{10}$

Kehren wir nun zu den Hegelschen Charakteristiken der bürgerlichen Gesellschaft zurück, beispielweise in seinen Grundlinien der Philosophie des Rechts. Eine konkrete menschliche Person, die „sich als besondere Zweck ist, als ein Ganzes von Bedürfnissen und eine Vermischung von Naturnotwendigkeit und Willkür, ist das eine Prinzip der bürgerlichen Gesellschaft (...)."11 Das zweite Prinzip dieser Form der Vergesellschaftung bildet die Notwendigkeit der Vermittlung der Befriedigung von Bedürfnissen in der Anerkennung durch eine andere Person und durch eine Zusammenarbeit mit ihr. Das heißt: durch andere Menschen, die mir gegenüber als Personen hervortreten und von mir die Anerkennung erwarten. Infolge dessen

8 Ebd., § 525: „In die Besonderheit der Bedürfnisse scheint die Allgemeinheit zunächst so, dass der Verstand an ihnen unterscheidet und dadurch sie selbst wie die Mittel für diese Unterscheide ins Unbestimmte vervielfältigt und beides immer abstrakter macht; diese Vereinzelung des Inhalts durch Abstraktion gibt de Teilung der Arbeit".

9 Ebd., § 526: „Die damit zugleich abstraktere Arbeit führt (...) durch ihre Einförmigkeit (...) zur Beschränkung auf eine Geschicklichkeit (...). Die Geschicklichkeit selbst wird auf diese Weise mechanisch und bekommt die Fähigkeit, an die Stelle menschlicher Arbeit die Maschine treten zu lassen".

10 Insofern darf man sagen, dass Hegel die marxistisch geprägte Kritik des Imperialismus antizipiert, obwohl er weder die von Marx entwickelte Entfremdungslehre noch dessen Werttheorie teilt.

11 Hegel, Grundlinien der Philosophie des Rechts, Werke in 20 Bänder, Suhrkamp, Frankfurt 1986, § 182 . 
„(D)er selbstsüchtige Zweck in seiner Verwirklichung, so durch die Allgemeinheit bedingt, begründet ein System allseitiger Abhängigkeit, daß die Substanz und das Wohl des Einzelnen und sein rechtliches Dasein in die Substanz, das Wohl und Recht aller verflochten, darauf gegründet und nur in diesem Zusammenhange wirklich und gesichert ist". ${ }^{12}$

So eine Entzweiung und Vermittlung des Besonderen und des Allgemeinen macht die Annahme einer Abstrakten, immer gleichen, reinen Subjektivität in der Rolle des Ausgangspunktes einer deskriptiven Gesellschaftstheorie zu einem fragwürdigen Unternehmen. Der individualistische Standpunkt, charakteristisch für die bürgerliche Gesellschaft wird damit aber gar nicht aufgehoben. Das tabula-rasa-Individuum wird lediglich mit einem geistigen Inhalt gefüllt. Denn in der Individualität eines so begriffenen Individuums hat ihr natürlicher Egoismus seinen Platz. Deswegen stellt Hegel folgendes fest: „Die bürgerliche Gesellschaft bietet in diesen Gegensätzen und ihrer Verwicklung das Schauspiel ebenso der Ausschweifung, der Elend und des beiden gemeinschaftlichen physischen und sittlichen Verderbens dar."13

Die obigen Zitate zeigen meines Erachtens ganz klar, wie unbegründet solche Hegelinterpretationen sind, die in der bürgerlichen Gesellschaft ein ausgezeichnetes, grundlegendes Sittlichkeitselement sehen. Die bürgerliche Gesellschaft ist zwar ein lebensnotwendiger Teil der modernen Sittlichkeit, so einer aber, der für diese Sittlichkeit immer auch eine Bedrohung bzw. eine Gefahr darstellt. Glücklicherweise gibt es ein Heilmittel gegen diese ,sittlich verderbende" Herausforderung seitens der bürgerlichen Gesellschaft: Hegel lässt keinen Zweifel darüber, dass es nur in der Staat zu suchen sei. „Die Individuen sind als Bürger dieses Staates Privatpersonen, welche ihr eigenes Interesse zu ihrem Zwecke haben. Da dieser durch das Allgemeine vermittelt ist, das ihnen somit als Mittel erscheint, so kann er von ihnen nur erreicht werden, insofern sie selbst ihr Wesen, Wollen und Tun auf allgemeine Weise bestimmen und sich zu einem Gliede der Kette dieses Zusammenhangs machen“, schreibt Hegel in Grundlinien der Philosophie des Rechts. ${ }^{14}$

Es ist selbstverständlich, dass gerade diese individuellen und egoistischen Interessen, verstärkt durch das dynamisch wachsende System der Bedürfnisse, zum Antriebsmotor der kapitalistischen Wirtschaft werden. Der Regulierung und der Effektivitätssteigerung dieses Wirtschaftssystems dient seine Justiz. Im Ansatz erscheint in ihm auch das ethische Moment, und zwar in der Gestalt der Polizei und der Korporation, die ,die Besorgung des besonderen Interesses

12 Ebd., $§ 183$.

13 Ebd., § 185 (die Hervorhebung von mir - AP)

14 Ebd., § 187. 
als eines Gemeinsamen“ möglich machen. ${ }^{15}$ Das bedeutet, dass die bürgerliche Gesellschaft imstande ist, sich für die Durchführung ihres Fortbestehens zu organisieren; es ist aber nicht dieses Niveau der Selbstverwaltung, das das ganze, organisch gedachte Leben einer Gesellschaft umfassen könnte. Bürgerliche Gesellschaft ist also kein Synonym für die „ganze“ Gesellschaft im Sinne eines Volkes oder einer Nation; es ist ein Name für die kapitalistische Form der Herstellung (Produktion) bzw. Lebensreproduktion. Eben darin ist aber für Hegel die menschliche Kreativität untergebracht, die jedoch oft über die natürliche Bedürfnisse der Menschen herausgeht und künstliche Bedürfnisse erschafft, nur um für die produzierten oder produzierbaren Waren einen Absatzmarkt zu finden. Hegel fasst es in folgenden Worten:

„Indem im gesellschaftlichen Bedürfnisse, als der Verknüpfung von unmittelbaren oder natürlichen und vom geistigen Bedürfnisse der Vorstellung, das letztere sich als das Allgemeine zum Überwiegenden macht, so liegt in diesem gesellschaftlichen Momente die Seite der Befreiung, dass die strenge Naturnotwendigkeit des Bedürfnisses versteckt wird (...). Diese Befreiung ist formell, indem die Besonderheit der Zwecke der zugrunde liegende Inhalt bleibt. Die Richtung des gesellschaftlichen Zustandes auf die unbestimmte Vervielfältigung und Spezifizierung der Bedürfnisse, Mittel und Genüsse, welche, so wie der Unterschied zwischen natürlichem und ungebildeten Bedürfnisse, keine Grenzen hat, - der Luxus - ist eine ebenso unendliche Vermehrung der Abhängigkeit und Not (...)."16

Zum Inhaltlichen der Hegelschen Gesellschaftstheorie der bürgerlichen Gesellschaft gehört auch, dass er darin bekanntlich drei maßgeblichen Menschengruppen auszeichnet. Das Kriterium der Unterteilung ist die Art der von ihnen geleisteten Arbeit. Diese Unterteilung in drei Stände: ein substanzieller, ein formeller und ein allgemeiner, heute vielleicht infolge der Entwicklung der Arbeitsteilung nicht mehr aktuell, spielt eine wichtige Rolle in der Hegelschen Auffassung der Demokratie. Diesen Aspekt müssen wir aber hier beiseite legen, vielleicht nur mit einer kurzen Bemerkung versehen, dass diejenige Interpreten, die in Hegel einen revisionistischen Antidemokraten sehen wollen völlig falsch liegen, auch wenn er mit der Idee einer rein ,arithmetischen“ Demokratie nicht sympathisiert.

Hegels Kritik der bürgerlichen Gesellschaft kann meines Erachtens als die Ausarbeitung der positiven und der negativen Entwicklungstendenzen der Moderne gelten. Trotz der Hervorhebung der Dynamisierung und der freiheitlichen Gestaltung des Lebens, die die Entstehung der bürgerlichen Gesellschaft mit sich bringt, betont Hegel ebenfalls die Bedrohungen für die Gestaltung der 
Sittlichkeit, die mit der Dominanz dieser Form der Vergesellschaftung einhergehen. Deshalb verankert er die bürgerliche Gesellschaft in dem Schema der Sittlichkeit, dessen Klammern die Familie einerseits und der Staat andererseits sind.

Anthropologisch gesehen ist Hegel im Klaren, dass die Entstehung der bürgerlichen Gesellschaft zwar wichtige innovative Kräfte ins Leben ruft, andererseits aber durch den damit verbundenen Individualismus jede Gesellschaft atomisieren und zum Zerfall bringen kann. Daher kann seine politische Philosophie als Kritik des Kapitalismus gelten, eine andere aber als die seines Schülers Karl Marx. Wo Marx die Zerstörung des bisherigen Herrschafts- und Produktionssystems vorschlägt, samt dem Staat und der bürgerlichen Gesellschaft, weil er in den beiden die Quelle der vielschichtigen Entfremdung sieht, plädiert Hegel für die Sorge um das Gleichgewicht zwischen den das gemeinschaftliche Leben tragenden Säulen.

Für Hegel ist die bürgerliche Gesellschaft mit der Bevölkerung eines Staats nicht identisch. Diese Bevölkerung, oder besser gesagt: dieses Volk, ist nicht nur ein System der Bedürfnisse; es ist auch eine historische Gemeinschaft, in derer Fortbestehen das sittliche Element eine wesentliche Rolle spielt. Deshalb ist die moderne, monogame Familie, als soziale Institution, notwendig, um den Kindern, die in die Welt der Erwachsenen eintreten, die elementare Moralität einzuprägen. Deshalb ist der Staat notwendig, als eine Institution, die die Sittlichkeit auf einem höheren Niveau betreut, als die Institution, die sich nicht nur um das Recht, sondern auch um die Gerechtigkeit kümmern soll. ,Zunächst ist die Familie - schreibt Hegel - das substantielle Ganze, dem die Vorsorge für diese besondere Seite des Individuums sowohl in Rücksicht der Mittel und Geschicklichkeit, um aus dem allgemeinen Vermögen sich /etwas/ erwerben zu können, als auch seiner Subsistenz und Versorgung im Falle eintretender Unfähigkeit angehört. Die bürgerliche Gesellschaft reißt aber das Individuum aus diesem Band heraus, entfremdet dessen Glieder einander und anerkennt sie als selbständige Personen." ${ }^{17}$ Deswegen ruht auf der bürgerlichen Gesellschaft die Pflicht des weiteren Ausbaus der moralischen Basis, die die familiäre Erziehung hervorbrachte. Denn es ist die Familie (und die Korporation), die die ethische Quelle des Staates bildet. In einem eigenhändigen Notiz Hegels, das als Ergänzung zum $§ 255$ der Grundlinien der Philosophie des Rechts gemeint war, stellt Hegel fest: „Heiligkeit der Ehe und die Ehre in der Korporation sind zwei Momente, um welche sich die Desorganisation der bürgerlichen Gesellschaft dreht“. ${ }^{18}$

17 Ebd., § 238.

18 Vgl.: Zitierte Auflage, S. 396. 
Die Rolle dieser Heiligkeit kann von der bürgerlichen Gesellschaft nicht übernommen werden, denn sogar wenn die äußere (z.B. Frieden) und innere (z.B. gutes Recht) Bedingungen die Entwicklung begünstigen, ,vermehrt sich die Anhäufung der Reichtümer - (...) - auf der einen Seite, wie auf der andern Seite die Vereinzelung und Beschränktheit der besonderen Arbeit und damit die Abhängigkeit und Not der an diese Arbeit gebundenen Klasse, womit die Unfähigkeit der Empfindung und des Genusses der weiteren Freiheiten und besonders der geistigen Vorteile der bürgerlichen Gesellschaft zusammenhängt". 19

Die hier in Frage kommende Not führt zur Entstehung des Pöbels, die selbst für die bürgerliche Gesellschaft eine Gefahr darstellt. „Das Herabsinken einer großen Masse - führt Hegel aus - unter das Maß einer gewissen Subsistenzweise, die sich von selbst als die für ein Mitglied der Gesellschaft notwenige reguliert - (...) - bringt die Erzeugung des Pöbels hervor, die hinwiederum zugleich die größere Leichtigkeit, unverhältnismäßige Reichtümer in wenige Hände zu konzentrieren, mit sich führt. “20

Die letzte Formulierung scheint zu beweisen, dass Hegel sogar die Existenz der Ausbeutung annimmt, einer Situation, die durch Verarmung und Desorganisierung der Arbeitermasse möglich wird. Eine mildernde Intervention seitens der bürgerlichen Gesellschaft weist er jedoch zurück. Zu seiner Zeit war sie wahrscheinlich schwer möglich. Aber auch in unserer ist sie nur mit der Vermittlung des Staates vorstellbar, denn der Staat ist nicht nur Repräsentant einer konkreten Bevölkerung, sondern auch einer ethischen Idee, die jede historische Nation verwirklicht. Denn Staat ist eine „substantielle Einheit“ und „absoluter unbewegter Selbstzweck, in welchem die Freiheit zu ihrem höchsten Recht kommt, so wie dieser Endzweck das höchste Recht gegen die Einzelnen hat, deren höchste Pflicht es ist, Mitglieder des Staates zu sein.“21

Nicht die bürgerliche Gesellschaft, sondern der Staat ist - wie er sagt - ,die Wirklichkeit der konkreten Freiheit“, die darauf beruht, dass „die persönliche Einzelheit und deren besondere Interessen sowohl ihre vollständige Entwicklung und die Anerkennung ihres Rechts für sich (im System der Familie und der bürgerlichen Gesellschaft haben, als sie durch sich selbst in das Interesse des Allgemeinen teil übergehen, teils mit Wissen und Wollen dasselbe und zwar als ihren eigenen substantiellen Geist anerkennen und für dasselbe als ihren Endzweck tätig sind (...)."22 Der moderne Staat ist also eine Form der Vergesellschaftung, die einerseits dem Prinzip der Subjektivität bzw. der Persönlichkeit

19 Ebd., § 243

20 Ebd., § 244.

21 Ebd., § 258.

22 Ebd., § 260. 
zur vollen Entwicklung verhilft, andrerseits aber die Bindung der Individuen an das substantielle Ganze, die ihre geistige und materielle Existenz bedingt, aufrechterhält. Riskant ist also die Behauptung, die den Staat der bürgerlichen Gesellschaft unterordnet. In Grundlinien der Philosophie des Rechts stellt Hegel eindeutig fest, dass: „Gegen die Sphären des Privatrechts und Privatwohls, der Familie und der bürgerlichen Gesellschaft ist der Staat einerseits eine äußerliche Notwendigkeit und ihre höhere Macht, deren Natur ihre Gesetze sowie ihre Interessen untergeordnet und davon abhängig sind; aber andrerseits ist er ihr immanenter Zweck und hat seine Stärke in der Einheit seines allgemeinen Endzwecks und des besonderen Interesses der Individuen, darin, dass sie insofern Pflichten gegen ihn haben, als sie zugleich Rechte haben.“"23

Die Lehre, die wir, die Heutigen, aus seiner Auffassung der bürgerlichen Gesellschaft ziehen können, sieht etwa wie folgt aus: Hegel, der größte Philosoph des 19. Jahrhunderts, fasst sehr treffend den unaufhaltbaren Triumph des kapitalistischen Wirtschaftssystems auf, als einer modernen, dem Stand der zivilisatorischen Entwicklung der Menschheit entsprechenden Form der Befriedigung der Bedürfnisse, die infolge der wachsenden Autonomie der Individuen sich immer weiter entwickeln, vergrößern und verkomplizieren. Die Bedingungen so einer Befreiung der menschlichen Individualität zur Selbstverwirklichung schaffen gemeinsam die christliche Religion (mit ihrer Idee der Gleichheit aller Menschen, als Personen, vor Gott) und das römische Recht (mit seiner Idee der Rechtsperson). Diese Entwicklung bedeutet jedoch zugleich einen allmählichen Zerfall der alten Formen der Sittlichkeit, die von einer neuen Sittlichkeit ersetzt werden müssen. Denn ohne eine Sittlichkeit ist ein kohärentes Bestehen einer jeden Gesellschaft unvorstellbar und auf Dauer unmöglich.

Theorien eines Gesellschaftsvertrags lehnt Hegel strikt ab. Sie mögen für ihn zwar interessante Gedankenexperimente darstellen, die wirkliche Gestaltung einer Gesellschaft bilden sie aber nicht ab. Denn - und das, würde ich sagen, ist eine „echt hermeneutische“ Komponente im Denken Hegels - jede Gesellschaft hat eine - oft blutige - Geschichte ihrer Entwicklung hinter sich, eine Geschichte, die ihre Mitglieder maßgeblich mitgestaltet hatte. Das führt oft dazu, dass - wie er bemerkt - zwei geographisch nachbarliche Länder unterschiedliche Staatsverfassungen haben. ${ }^{24}$

In der Sorge ums Fortbestehen der ausbalancierten Triade: Familie - bürgerliche Gesellschaft - Staat sieht Hegel die Maßnahme gegen die Krankheiten des modernen, durch Kapitalismus geprägten, Lebens. Hegel scheint dabei ein - wie bereits erwähnt - durchaus hermeneutisch gesinnter Denker zu sein, für

23 Ebd., § 261.

24 Polen und Preußen zur Hegels Lebenszeit wären ein gutes Beispiel dafür. 
den der ,aufklärerische Abstraktionismus der reinen Vernunft“ bzw. des reinen Subjekts (was zu einem „Menschen ohne Eigenschaften“ führen müsste) ein gefährlicher Denkfehler bedeutet würde. Das relativiert sehr stark die Wahrheit einer Hegel zugeschriebenen Behauptung, der zufolge „moderne Staaten im Element der Individualisierung gründen“ ${ }^{25}$ Moderne Staaten begünstigen zwar die Individualisierung, hören aber nicht auf, das Frucht und zugleich der Schutz der historisch gebildeten kulturellen Gemeinschaft eines Volks zu sein, die in Hegels Augen die höchste Inkorporierung des Geistes darstellt.

Zum Schluss möchte ich kurz auf die Frage möglicher Aktualität der Hegelschen Theorie der Sittlichkeit eingehen. In der Perspektive der wachsenden Vereinheitlichung Europas, vor allem durch die EU, scheint seine Verteidigung der Rolle des Staates obsolet zu sein. Aus der Perspektive der moralischen Umwandlungen des westlichen Welt, vor allem in der 2. Hälfte des 20. Jahrhunderts, kann man auch seine Verteidigung der Familie als des Geburtsorts der primären Sittlichkeit als obsolet behandeln. Der Zerfall der Institution der Familie durch die Zulassung der gleichberechtigten homosexuellen Ehen scheint hier ein wichtiges Argument zu sein. Aber Argument wofür? Ob es ein Argument für die Rückständigkeit des Hegelschen Gesellschaftsbildes ist, werden wir erst in ein paar Hundertenjahren wissen können, wenn die Ehe und die Familie im heutigen Sinn total verschwinden. Ich habe hier erhebliche Zweifel, ob die Entwicklung, die wir gerade erfahren, einen echten Fortschritt bedeutet.

Die gleiche Skepsis gilt dem Verschwinden bzw. der erheblichen Schwächung des Staates. Der Zerfall von Sowjet Union, der Wiedergeburt von Litauen, Lettland, Georgien, etc. lässt sich vielleicht noch mit den Wille zur Befreiung von einem totalitären Regierungssystem erklären. Gilt diese Erklärung automatisch auch für den Zerfall der Tschechoslowakei? Und für die Balkankriege, die zum Zerfall eines Kunstproduktes namens Jugoslawien führten? All das sind Beweise dafür, dass der eigene Staat für viele Völker nach wie vor die beste Betreuung und effektivste Vertretung ihrer Nationalinteressen ist, und zwar nicht nur der materiellen, sondern auch derjenigen, die der geistiger Natur sind. Es entstehen hier ohne Zweifel viele wichtige Fragen und Probleme, die subtiler, hermeneutisch geprägter Natur sind, und die die Brüsseler Bürokratie meistens mit Zwang, Druck und Gewalt zu lösen versucht. Oft mit Erfolg.

Das 19. Jahrhundert gilt für viele als das Jahrhundert der Nationenbildungen. Die vermeintlichen Resultate dieser Entwicklung, nämlich die beiden Weltkriege (und viele kleinere Kriege in und außer von Europa), sollten den Beweis dafür darstellen, dass die Idee des Staates zu überwinden gilt, und zwar zugunsten einer internationalen Vereinigung, die die Bedeutung der Nationen

25 Die Formulierung stammt vom exzellenten polnischen Hegel-Forscher, Marek J. Siemek. Vgl. M. J. Siemek, Hegel i filozofia, Oficyna Wydawnicza, Warszawa 1998. 
und ihrer Staaten minimieren soll. Dennoch ist - erstens - die Idee, dass es die Nationen waren, die gegeneinander kämpften, schlicht und einfach falsch, denn es waren entweder die militarisierten Ideologien (Faschismus, Nationalsozialismus und Bolschewismus im 2. Weltkrieg) oder die multinationalen Imperien (Russland, Österreich-Ungarn, Preußen, Großbritannien usw. im 1. Weltkrieg). Eine normal sich entwickelnde Nation, die auf einem Territorium im Medium eigener Kultur gedeiht, hat an sich kaum Gründe, andere Nationen zu bekämpfen bzw. zu versklaven. Der Staat als Vertretung einer Kultur- und Sprachgemeinschaft hat - zweitens - eine tiefe hermeneutische Berechtigung. Als Europäer bzw. als Menschen überhaupt teilen wir zwar die Erzeugnisse der Zivilisation, die uns zugänglich sind (vor allem die technischen), unsere Identität - und damit auch unsere Differenz zu anderen - kommt aber größtenteils aus der kulturellen Überlieferung, aus der Kultur, in der wir aufwachsen, die letztendlich sprachlich (im breiten Sinne des Wortes) geprägt ist. Deshalb kann es nur einen hermeneutisch Unaufgeklärten wundern, dass auch im 21. Jahrhundert Basken, Katalanen oder Kurden den wichtigsten Faktor ihrer kultur-politischen Entwicklung in der Errichtung ihres eigenen Staates sehen. Daraus müssen wir lernen, dass die EU nichts mehr als eine Föderation der unabhängigen Staaten sein darf. Denn nur so haben wir die Chance - erstens eine echte - und nicht die künstlich aufgepresste - Identität zu gewinnen, und - zweitens - in verschiedenen Ländern unterschiedliche Lösungen für unsere Probleme auszuprobieren, womit wir unsere Erfahrung, als Quelle unserer historisch geprägten Vernunft, erheblich erweitern. Unsere untilgbare Vielheit ist unser größter Schatz.

Für Hans-Georg Gadamer war die Hegelsche Triade: „Familie - bürgerliche Gesellschaft - Staat" so selbstverständlich, dass er sie direkt im Anschluss an die Darstellung der Aristotelischen Ethik erwähnt, als ob sie schon zur Zeit des großen Griechen maßgeblich wäre. Der Heidelberger Hermeneutiker schreibt: „Gewiss ist jedermann abhängig von den Vorstellungen seiner Zeit und seiner Welt, aber weder folgt daraus die Legitimität des moralischen Skeptizismus noch auch die des technischen Manipulierens aller Meinungsbildung unter der Perspektive der politischen Machtausübung. Die Veränderungen, die in Sitte und Denkart einer Zeit Platz greifen und die insbesondere den Älteren den bedrohlichen Eindruck einer totalen Auflösung der Sitte zu geben pflegen, vollziehen sich auf einem ruhenden Grunde. Familie, Gesellschaft, Staat bestimmen die Wesensverfassung der Menschen, indem sich sein Ethos mit wachsenden Inhalten füllt." 26 .

26 H.-G. Gadamer, ,Über die Möglichkeiten einer philosophischen Ethik“, in: Gadamer, GW, B. 4, S. 188. (Hervorhebung - AP). 
Weder der Staat noch die Familie (und ihr Kern: die Ehe) haben in der bürokratischen und linksgeprägten Machtstrukturen der EU einen guten Ruf. Die langsame, oft versteckte Übertragung der Machtkompetenzen von den Einzelstaaten auf die Europäische Kommission und auf das Europaparlament oder der Kampf der Union-Bürokratie um die Gleichstellung der Hetero- und Homoehe stellen diese These unterm Beweis. Die Frage, die sich daraus ergibt, lautet: was wird unsere sittliche Substanz bilden, wenn die die Liebe und das Vertrauen spendende Kraft der Familie und die kulturelle Verankerung schaffende Kraft des Staates ausgelöscht werden? Ein allgemein europäisches Bewusstsein, gestützt auf einem Verfassungspatriotismus, bleibt nach wie vor eine Illusion. ${ }^{27}$ Die Einführung der gemeinsamen Währung wird es, gegen alle Hoffnungen, nicht schaffen. Ohne Etablierung einer gemeinsamen ganzeuropäischen Sprache, in der alle unsere Kinder künftig erwachsen würden, ohne Einführung einer gemeinsamen historischen Bildung, kann sich diese Idee in vorstellbarer Zeit nicht verwirklichen. Das ist auch eine Lehre, die wir aus der hermeneutisch-anthropologischen Lektüre Hegels ziehen können, ja - sogar müssen.

27 Neulich musste es sogar der ehemalige Befürworter dieser Idee, Jürgen Habermas, zugegeben haben. 
